\title{
Analysis of PLC transmission characteristics of urban distribution network
}

\author{
Jia-hao Feng ${ }^{1, a}$, Dong Wang ${ }^{2, a}$, Yong Li ${ }^{3, b}$, Dong-jie Wang ${ }^{4, b}$, Xue-jun Qiao ${ }^{5, b}$ \\ ${ }^{a}$ (Department of Electrical and Electronic Engineering, North China Electric Power University, \\ Baoding 071003, Hebei Province, China) \\ ${ }^{b}$ (Baoding electric power company, Hebei Province, China) \\ ${ }^{a}$ Email: 949890347@qq.com, ${ }^{b}$ Email: 863765114@qq.com
}

\begin{abstract}
Keywords: Distribution network, Narrow Band Power Line Communications Technology (NB-PLC), hybrid lines, Transmission characteristics .

Abstract: $10 \mathrm{kV}$ distribution network topology is complex, along the lines there are many connected power distribution transformer and branch lines, and the lines are mostly hybrid cases. Its channel transmission characteristics are complex and variable, which has caused great difficulties to the high rate $N B-P L C$ technology frequency selection. Based on urban $10 \mathrm{kV}$ distribution network topology, it emphatically analyzes the changes in the channel transmission characteristics from the carrier source to the receiving point in hybrid cases and influence rules of the length of main trunk line, the length and number of branch line and the location and quantity of distribution transformer on the channel transmission characteristics, which provide reference basis for the modulation mode and frequency selection of high rate $N B-P L C$ technology.
\end{abstract}

\section{Introduction}

At present, China's distribution network planning determines upon a combination of optical fiber, carrier and wireless variety of ways in distribution communication system, but in the actual construction, in terms of optical fiber communication, there are some difficulties at the level of expensive cable laying and maintenance workload. At the same time, there are some problems of low reliability in wireless communication. And power line carrier communication saves construction investment by using ready-made distribution network as a channel. With the rapid development of NB-PLC (OFDM) technology, Narrow Band Power Line Communications Technology $(N B-P L C)$ based on multiple carrier modulation method has become the current hot issue in the universal intelligent distribution network communication technology. At the same time, IEEE (IEEE Std. 1901.2-2013) standard for smart grid applications has just promulgated. High speed $N B-P L C$ technology has already overcome many inherent shortcomings of the traditional $P L C$, with a higher anti-interference ability, adaptive ability and networking flexibility. Therefore, the research on the PLC communication technology of the new generation medium voltage power distribution network is the basic work of building the intelligent distribution network and realizing the energy Internet.

Because the distribution network of urban power distribution network is a hybrid line, and the distribution network topology is complex and changeable, the signal is prone to refraction and reflection, leading to signal frequency selective fading.Therefore, the analysis of the factors that influence the channel characteristics is the essential prerequisite and the important basis for the application of the power line carrier communication technology in the smart distribution grid. This paper is to analyze the factors that influence the channel characteristics of the carrier communication in the $10 \mathrm{kV}$ city distribution network. 


\section{Influence factors of distribution network carrier communication channel}

The typical structure of urban distribution network is as follows:

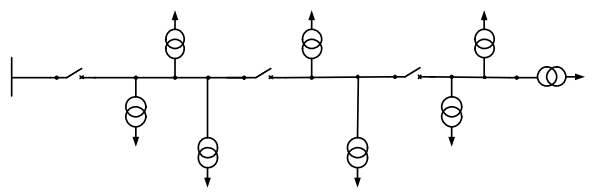

Fig.1. Topology of urban distribution network

It can be seen that the network topology of city distribution network is more complex than the power transmission network, there are many connected distribution transformers along the line; and many branch lines are connected to trunk lines and the main line and branch line have both the overhead lines and cable lines, so cable lines and overhead lines are all in existence and the complexity of the carrier communication channel are taking shape. In order to realize the automation of distribution network, the research on the impact of these factors on carrier communication must be conducted. This paper mainly analyzes the following factors:

\section{Impedance matching problem between the components}

(1).Source impedance matching. Power resistance is equal to the characteristic impedance of the transmission line.

(2).Characteristic impedance matching.

the typical parameters of the characteristic impedance of overhead line are $200 \sim 400 \Omega$; the typical parameters of cable line characteristic impedance of cable lines are $20 \sim 40 \Omega$. For the two connected line, the reflection

coefficient can be expressed as: $\Gamma_{12}=\frac{Z_{c 2}-Z_{c 1}}{Z_{c 1}+Z_{c 2}} ; Z_{c 1}, Z_{c 2}$ are the characteristic impedance of each line (representing the reflection coefficient from line1to line 2).

(3).Load impedance matching. Load impedance matching is the impedance of the loadand the characteristics of the transmission line.

Effect of power distribution transformer.

For the $10 \mathrm{kV}$ distribution transformers, theinput impedance of high voltage side is capacitance.The impedance value decreases with the increase of the frequency and with the increase of the capacity, the impedance value becomes smaller [1,2].

\section{Effect of branch line.}

The existence of a branch line is equivalent to avariable impedanceon the main line of the power distribution, the size of the impedance relates to the length of thebranch line, the position and the nu mber of the transformer on the branch line. The impedanceis small, the shunt is large and the loss is large.

\section{Carrier communication channel models}

\section{Transmission line model}

The majority of the current $10 \mathrm{kV}$ urban distribution network line is the hybrid line of overhead lines and cables. In consideration of different parameters and different wave impedance, the signal transmission characteristics of them are disparate. In the analysis of the carrier communication channel modeling, the length parameters per unit of overhead lines and cable lines should be calculated $[3,4,5]$, finding out respective propagation constant $\gamma$ and characteristic impedance $Z_{c}$.

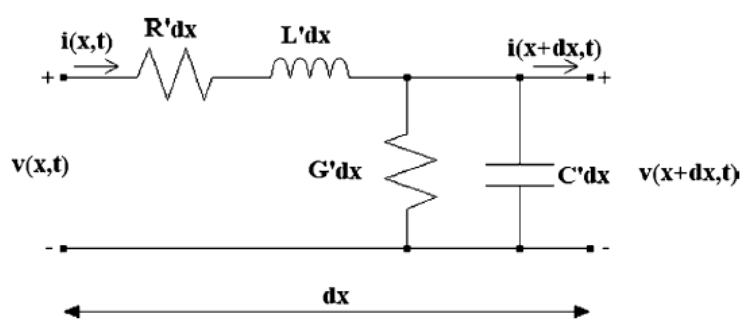

Fig. 2 .Power line transmission model 
The voltage and current at different positions are also different, in the presence of electric resistance, inductance and capacitance conductance. High frequency current can be described by the uniform transmission line equation along the transmission line. By telegraph equation:

$$
\left\{\begin{array}{l}
\frac{\partial v(x, t)}{\partial x}+R^{\prime} i(x, t)+L^{\prime} \frac{\partial i(x, t)}{\partial x}=0 \\
\frac{\partial i(x, t)}{\partial x}+G^{\prime} v(x, t)+C^{\prime} \frac{\partial v(x, t)}{\partial x}=0 \\
R^{\prime}, \quad L^{\prime}, \quad G^{\prime}, \quad C^{\prime} \text { are the per unit length }
\end{array}\right.
$$

parameter. The parameters to describe a transmission are line the propagation constant $\gamma$ and the characteristic impedance $Z_{c}$.

$$
Z_{C}=\sqrt{\left(R^{\prime}+j \omega L^{\prime}\right) /\left(G^{\prime}+j \omega C^{\prime}\right)} \quad \text { (2) } \gamma=\alpha+j \beta=\sqrt{\left(R^{\prime}+j \omega L^{\prime}\right)\left(G^{\prime}+j \omega C^{\prime}\right)}
$$

$10 \mathrm{kV}$ distribution network in China is commonly used in different cross sections of overhead insulated lines (JKLYJ) and cable lines (YJV22). The characteristic impedance of the two is shown in table 1.

Table1.characteristic impedance:

\begin{tabular}{|l|l|l|l|}
\hline \multicolumn{2}{|c|}{ YJV22 } & \multicolumn{2}{c|}{ JKLYJ } \\
\hline $\begin{array}{l}\text { Cross-sectio } \\
\text { ns(mm2) }\end{array}$ & $Z_{c}(\Omega)$ & $\begin{array}{l}\text { Cross-secti } \\
\text { ons }(\mathrm{mm} 2)\end{array}$ & $Z_{c}(\Omega)$ \\
\hline 50 & 41.85 & 70 & 284 \\
\hline 70 & 38.55 & 120 & 275 \\
\hline 120 & 32.32 & 240 & 261.8 \\
\hline 240 & 26.45 & & \\
\hline 300 & 24 & & \\
\hline
\end{tabular}

\section{The transformer model}

City power distribution network trunk line is mostly distributed in the load intensive areas, there are also many connected distribution transformers along the line. From a great deal of literature [1,2], the research results that the low voltage of the transformer is to open circuit or short circuit, the measurement of the high voltage side impedance is basically unchanged.Thus, the distribution and load can be replaced by an equivalent highfrequency model. With the increase of the frequency, the low frequency $\mathrm{T}$ circuit will no longer be used in high frequency. Therefore,this paper adopts the high frequency equivalent model of transformer three capacitor, as shown in Fig.3.In the case of the phase $\mathrm{A}, C_{1}$ and $C_{2}$ represent the turn-to-turn capacitances respectively of the primary and the secondary. The capacitance $C_{12}$ is the capacitance between windings. The magnetizing impedance is modelled thanks to a resistance $\left(R_{m}\right)$ with an inductance in parallel $\left(L_{m}\right) . R_{1}, R_{2}^{\prime}, L_{1}, L_{2}^{\prime}$ represent the leakage resistances and inductances.

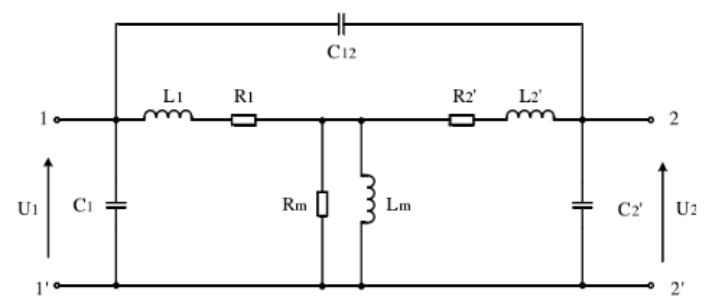

Fig.3. High frequency model of power transformer

Fig. 4 shows the input impedance from the high frequency equivalent model of a transformer. From the figure, the frequency is $70 \sim 500 \mathrm{kHz}$, the input impedance of this model is capacitance and the impedance value is reduced with the increase of frequency. 

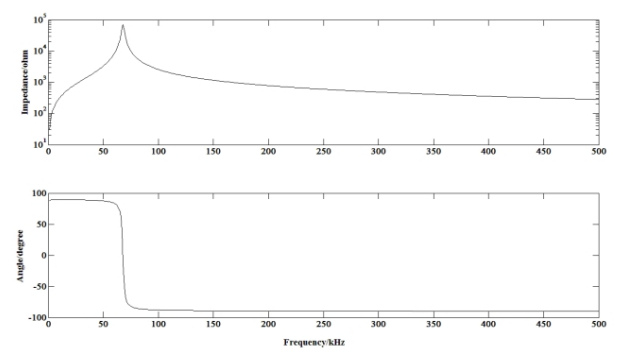

Fig.4. Input impedance and phase frequency characteristic (transformer: $10 \mathrm{kV} / 0.4 \mathrm{kV}$ and $100 \mathrm{kVA}$ )

In the paper, a mathematical model is built to analyze the channel characteristics on the basis of the modeling method[6] .

\section{$10 \mathrm{kV}$ carrier channel characteristics analysis}

\section{Different length of trunk line}

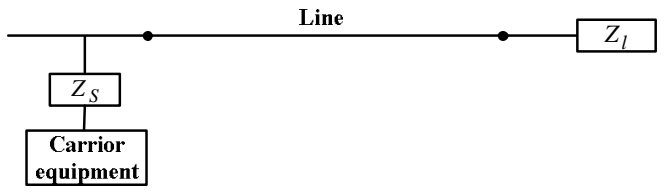

Fig.5. Topology of simple power line (Note: take $Z_{l}=75 \Omega, \quad Z_{s}=50 \Omega$ )

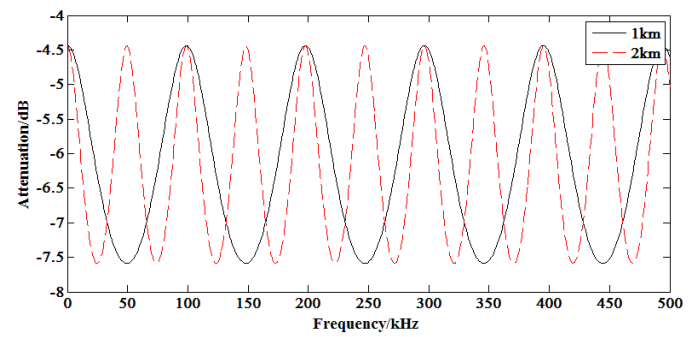

Fig.6. Voltage amplitude frequency characteristics of YJV22-300 $\mathrm{mm}^{2}$

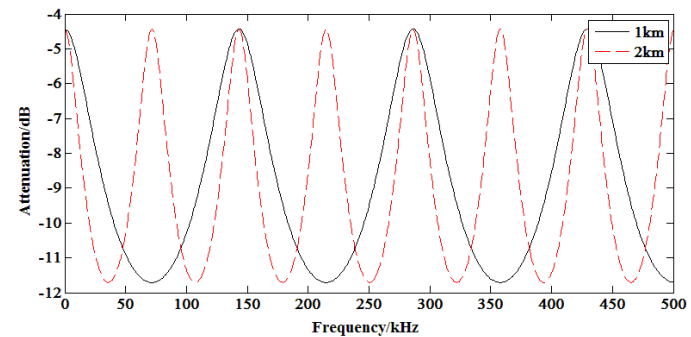

Fig.7. Voltage amplitude frequency characteristics of JKLYJ-120 $\mathrm{mm}^{2}$

Fig.13 illustrates that with the increase in the length of the branch line, the amplitude of the signal attenuation magnifies, at the same time the number of the peaks and valleys of attenuation is increasing significantly. Changingthe linking position of the branch line, the attenuation characteristics remain essentially unchanged.

Same type line of different cross sections

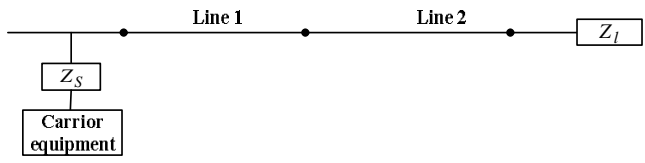

Fig.8. Topology of simple power line (Length of the line 1 or 2 is $1 \mathrm{~km}$ ) 


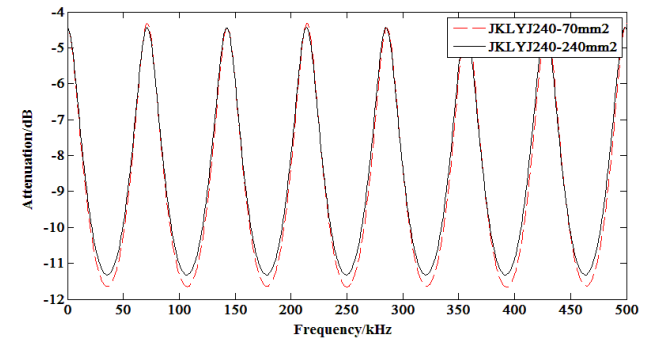

Fig.9. Voltage amplitude frequency characteristics of overhead line with different cross sections

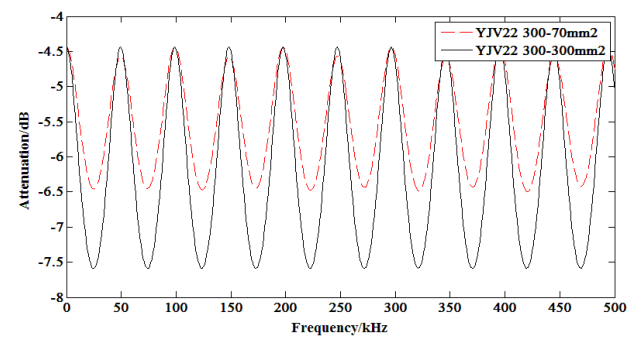

Fig.10. Voltage amplitude frequency characteristics of cable line with different cross sections

Fig.9 and Fig.10 tell that for the same type of overhead line, the value of the peak and valley of the attenuation, the number and the attenuation frequency are basically unchanged; For the same type of cable line with different cross sections, the number of the peaks and valleys of the attenuation and the attenuation frequency are basically unchanged, but the valley of the attenuation of has bigger value.

\section{Hybrid of overhead line and cable}

In Fig. 11, the trunk line is composed of overhead line by JKLYJ-120 $\mathrm{mm}^{2}, 1 \mathrm{~km}$ and cable by YJV22-70 $\mathrm{mm}^{2}, 1 \mathrm{~km}$.

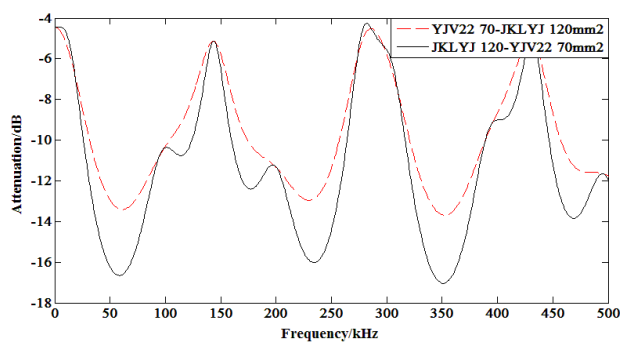

Fig.11. Voltage amplitude frequency characteristics of mixed line

Fig.11 shows the channel characteristics of the hybrid line. From the graph, comparing the signal from the source - the overhead line- cable to the signal from the source-cable - the overhead line, the attenuation of the former is greater than the one of the latter. And due to the large impedance difference of the mixed line, the attenuation of the signal transmission is greater than that of the same model line, which is disadvantageous for signal transmission in the hybrid line.

\section{Change length and the number of branches}

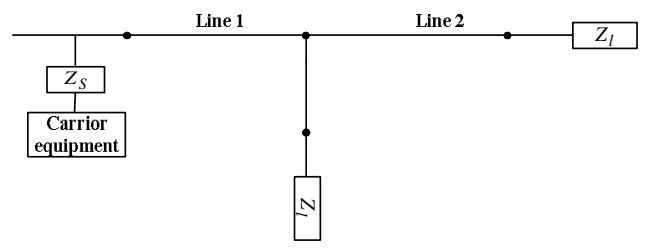

Fig.12. Topology of single branch

In Fig. 12, the trunk line is JKLYJ-120, 4km; branch line (JKLYJ-70)is connected to the midpoint of the trunk line. 


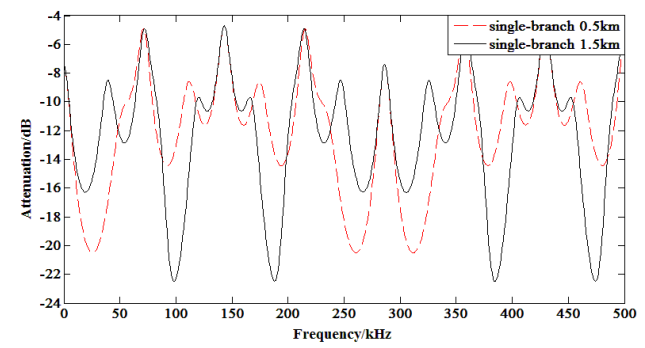

Fig.13. Voltage amplitude frequency characteristics of single branch

Fig.13 illustrates that with the increase in the length of the branch line, the amplitude of the signal attenuation magnifies, at the same time the number of the peaks and valleys of attenuation is increasing significantly. Changingthe linking position of the branch line, the attenuation characteristics remain essentially unchanged.

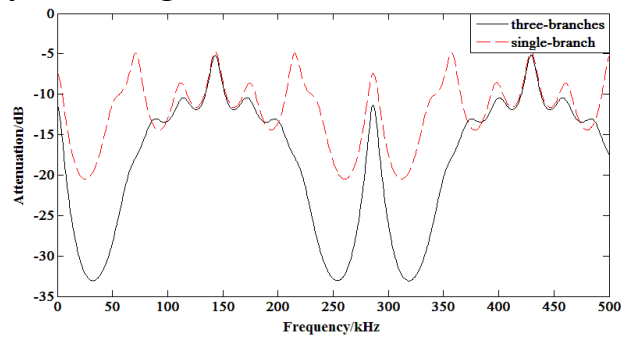

Fig.14. Voltage amplitude frequency characteristic of single branch and three branches

Fig.14 is the comparison chart that shows the channel characteristics of the three-branch lines and single branch line respectively. According to this figure, the following conclusion can be seen: in the increase in the number of branches, the attenuation amplitude increases significantly.

\section{Power distribution transformers}

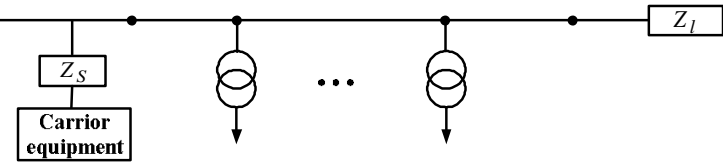

Fig. 15.Topology of multiple load transformers (The trunk line is JKLYJ-120 $\mathrm{mm}^{2}, 4 \mathrm{~km}$.)

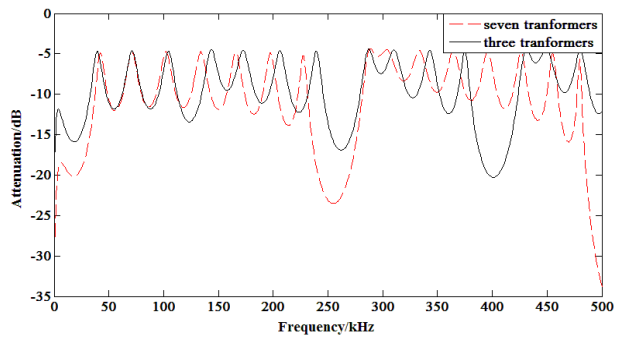

Fig.16. Voltage amplitude frequency characteristics of multiple load transformers

With the increase of the number of power distribution the channel transmission characteristics indicate apparent frequency selective

fading, and the attenuation amplitude is significantly increasing; and the number of distribution transformers is different, and the number of selective attenuation s also differs, with the increase of the number of the transformers, the number of selective fading valleys accordingly reduce, attenuation amplitude is on the rise.

Multiple branches and load transformers

Many power distribution transformers and multiple branches are connected along the urban distribution network. The network topology is complex and changeable. Fig. 17 shows the topology of multiple branches and load transformers distribution network. 


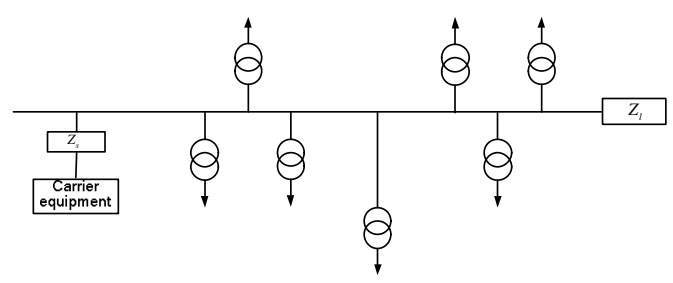

Fig.17. Topology of multiple branches and load transformers (The trunk line is JKLYJ-120 $\mathrm{mm}^{2}, 4 \mathrm{~km}$; the length of three branches (JKLYJ-70 $\mathrm{mm}^{2}$ ) is $0.5,0.5,1 \mathrm{~km}$.)

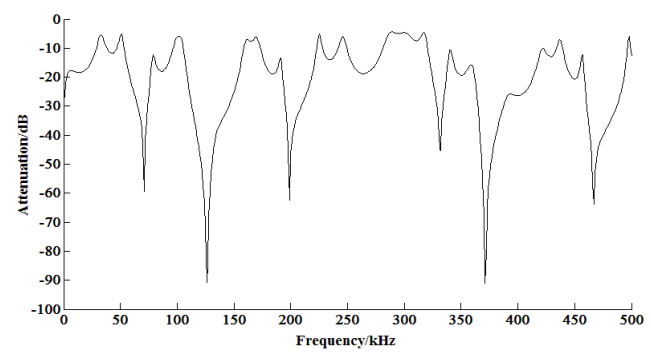

Fig.18. Voltage amplitude frequency characteristics of multiple branches and load transformers

According to the topology structure of the channel model, the signal transmission characteristics are obtained as shown in Fig. 18. It can be seen that, for distribution network, the number of branch lines, load transformers would affect the signal transmission characteristics. To some extent, in practical application, the best signal frequency is selected according to the transmission characteristics.

\section{Conclusions}

In this paper, some common phenomena can be found, and it draws the following conclusions by the simulation analysis of the PLC channel characteristics of the distribution network.

(1).The increase in trunk line, the numerical of the attenuation peak and valley is basically unchanged, and the number of the attenuation peak and valley increases with the increase of the length of the line.

(2). The attenuation of the signal transmitted in the mixed line is greater than the one in the same type of line, which is unfavorable for the signal transmission on the mixed line.

(3).With the increase of the number of distribution transformers, the channel transmission characteristics indicate apparent frequency selective fading, and the attenuation of the amplitude is significantly increasing.

(4).With the rise of length of branch line ,the number of peaks and valleys of the attenuation increase obviously, and the attenuation amplitude is greatly on the rise with the increase of the number of branches.

\section{References}

[1] Hemminger R C,Gale L J, Amoura F, et al.The effect of distribution transformers on distribution line carrier signals[J].IEEE Transactions on Power Delivery, vol.PWRD-2,NO.1,pp.36-40,Jan.1987.

[2] IEEE Communications Society, IEEE standard for low-frequency(less than $500 \mathrm{kHz}$ ) narr ow band power line communications for smart grid applications[S]. New York:March 20 13.

[3] Antonio Cataliotti, Alfred Daidone, etal. Power Line Communication in Medium Voltage Systems: Characterization of MVCables[J].IEEE Transactions on Power Delivery,vol.23, NO.4,pp.1896-1902,Oct.2010.

[4] Amir Mehdi Pasdar, Yilmaz Sozer Iqbal Husain. Novel Method for Real Time Overhead Power Line Segments High Frequency impedance Measurement Based on Signal Injection[C]// IEEE 
Applied Power Electronics Conference and Exposition (APEC). Long Beach, CA, USA, 2013: 2192 - 2197.

[5] Fabio Gianaroli, Alessandro Barbieri, etal.A novel approach to power-line channel modeling [J].IEEE Transactions on Power Delivery, vol.25,NO.1, pp.132-139,Jan.2010.

[6] Cai Wei, Le Jian, Liu Kaipei, et al.A channel modeling technology for the medium-voltage power-line carrier in smart power distribution grid[J] .Proceedings of the CSEE, 2012, 32(28):150-156(in Chinese). 